$$
\text { CONE- } 961202-59
$$

Paper- Invited Session

Defects in Electronic Materials, MRS Meeting, December 2-6, 1996

"The submitted manuscript has been authored

by a contractor of the U.S. Government under

contract No. DE-AC05-960R22464.

Accordingly, the U.S. Government retains

nonexclusive, royalty-free license to publish or

reproduce the published form of this

contribution, or allow others to do so, for U.S.

Government purposes."

है

JAN 283997

OSTI

\title{
COOPERATIVE CHEMICAL REBONDING IN THE SEGREGATION OF IMPURITIES IN SILICON GRAIN BOUNDARIES
}

A. Maiti, M. F. Chisholm, S. J. Pennycook, and S. T. Pantelides

\author{
SOLID STATE DIVISION \\ OAK RIDGE NATIONAL LABORATORY \\ Managed by \\ LOCKHEED MARTIN ENERGY RESEARCH CORP.
}

Under

Contract No. DE-AC05-960R22464

With the

U. S. DEPARTMENT OF ENERGY

OAK RIDGE, TENNESSEE

December 1996

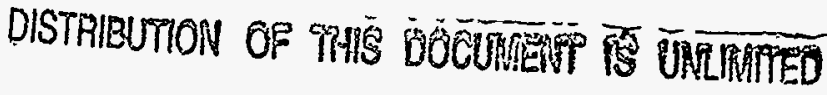<smiles>CCCCCCCC</smiles> 


\section{DISCLAIMER}

This report was prepared as an account of work sponsored by an agency of the United States Government. Neither the United States Government nor any agency thereof, nor any of their employees, make any warranty, express or implied, or assumes any legal liability or responsibility for the accuracy, completeness, or usefulness of any information, apparatus, product, or process disclosed, or represents that its use would not iniringe privately owned rights. Reference herein to any specific commercial product, process, or service by trade name, trademark, manufacturer, or otherwise does not necessarily constitute or imply its endorsement, recommendation, or favoring by the United States Government or any agency thereof. The views and opinions of authors expressed herein do not necescarily state or reflect those of the United States Government or any agency thereof. 


\section{DISCLAIMER}

Portions of this document may be illegible in electronic image products. Images are produced from the best available original document. 


\title{
COOPERATIVE CHEMICAL REBONDING IN THE SEGREGATION OF IMPURITIES IN SILICON GRAIN BOUNDARIES
}

\author{
A. Maiti, ${ }^{1,2}$ M. F. Chisholm, ${ }^{1}$ S. J. Pennycook, ${ }^{1}$ and S. T. Pantelides 1,2 \\ 1Solid State Division, Oak Ridge National Laboratory, Oak Ridge, TN 37831 \\ 2Department of Physics and Astronomy, Vanderbilt University, Nashville, TN 37235
}

\begin{abstract}
With $a b$ initio calculations we show that the experimentally observed large segregation energies of As at $\mathrm{Si}$ grain boundaries can be explained by the formation of isolated dimers or ordered chains of dimers of threefold-coordinated As along the cores of grain boundary dislocations. We also find the intriguing possibility that As segregation may drive structural transformation of certain grain boundaries. Recently we have obtained the first atomic-resolution STEM images of As in a Si grain boundary, consistent with the formation of As dimers. Segregation energy of As dimers was found to be significantly higher in isolated dislocation cores, where larger site-variation in strain than in grain boundaries lead to further lowering of the electronic levels of As deep into the bandgap.
\end{abstract}

\section{INTRODUCTION}

Polycrystalline semiconductors are used in microelectronics. Dopants, which control the electrical properties of these materials, are known to segregate in grain boundaries in electrically inactive configurations [1-4]. The fraction of dopants in the grain boundaries is governed by the segregation energy, i.e., the energy difference between a dopant atom in the grain boundary and a dopant atom in the bulk crystal. For the specific case of As segregation in Si and Ge grain boundaries, experimental values of the segregation energies range from 0.41 to $0.65 \mathrm{eV}[1-4]$.

Clearly, it would be very important for technologists to know if the large segregation energy is an intrinsic property of a defect-free grain boundary or is caused by defects that could, in principle, be avoided. Experiment $[5]$ and theory $[6,7]$ have established that tilt grain boundaries in undoped $\mathrm{Si}$ and $\mathrm{Ge}$ rebond so that all host atoms are fourfold-coordinated. The only first-principles theoretical study of segregation was reported recently by Arias and Joannopoulos [8]. These authors examined the segregation energies of isolated As atoms placed at different substitutional sites in a Ge grain boundary and found values only of order $0.1 \mathrm{eV}$. They proposed that the observed segregation energies are likely to arise from As atoms bound to steps or other defects. No calculations were pursued to explore such possibilities.

\section{MOTIVATION}

The motivation for the present work was the recognition that the observed large segregation energies may occur in defect-free grain boundaries because As atoms achieve their preferred threefold coordination, as they are known to do in amorphous $\mathrm{Si}$ and $\mathrm{Ge}$ [9]. Simple bond counting suggests that, if a single As atom were to achieve threefold coordination in a Si or Ge grain boundary, at least one $\mathrm{Si}(\mathrm{Ge})$ atom would have to have odd coordination ( 3 or 5), which is energetically costly. Clearly, threefold-coordinated As atoms would be far more likely if they were incorporated in a grain boundary in a 
cooperative manner, at least two at a time. The simplest possibility would be two As atoms at nearest neighbor sites. The As atoms might relax away from each other so that the "bond" between the As atoms is broken. In this way, each As atom attains its preferred threefold-coordination, with all the Si atoms fourfold-coordinated [10].

\section{COMPUTATIONAL PROCEDURE}

The calculations were based on density functional theory [11] with local exchange-correlation energy as parametrized by Perdew and Zunger [12]. The atomic cores are represented by non-local, norm-conserving pseudopotentials of the Kerker type [13] in a separable Kleinmann-Bylander form [14], and defined on a real-space grid [15]. The integration over the Brillouin Zone was performed using two special k-points chosen according to the Monkhorst-Pack scheme [16]. The electronic wavefunctions were expanded in a plane wave basis set with an energy cutoff of $150 \mathrm{eV}$, verified to yield accurate lattice constant and bulk modulus for the pure crystal. For each geometry, the electronic wavefunctions were first relaxed by the conjugate gradient scheme of Payne, et al. [17], until they reached a local minimum (the Born-Oppenheimer surface). The ions were then moved according to the Hellman-Feynman forces until the largest force on any ion in any direction was less than $0.08 \mathrm{eV} / \mathrm{A}$.

\section{SEGREGATION AT A $\Sigma=5\{310\}<001>$ TILT BOUNDARY}

\section{Relaxed Structures}

For simplicity and computational feasibility we first considered the symmetric $\Sigma=5$ tilt boundary in $\mathrm{Si}$, which is parallel to the $\{310\}$ plane of the original crystalline lattice and has the tilt axis parallel to the $<001>$ direction. It has a minimum periodicity of one conventional lattice parameter $(a-5.431 \AA)$ in the $<001>$ direction and a periodicity of a $\sqrt{5 / 2}=8.587 \AA$ in the direction perpendicular to the $<001>$ axis. The details of the supercell used for our calculations are described in Ref. 10. The relaxed ground-state structure, which we call GB1, is shown in Fig. 1 in a projection perpendicular to the tilt

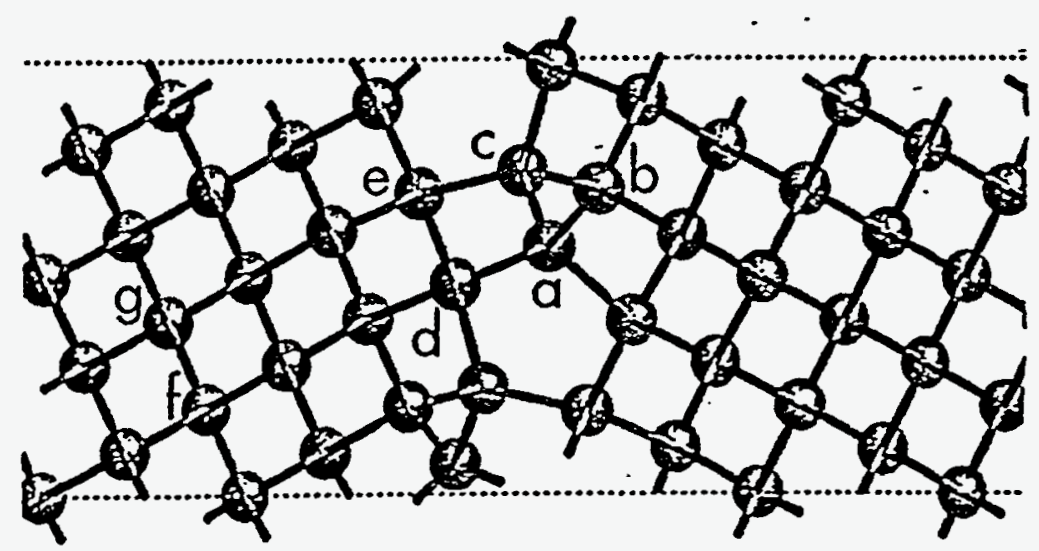

Fig. 1. Projection (normal to the tilt axis) of the relaxed structure of a $\Sigma=5\{310\}<001>$ symmetric grain boundary of Si in its ground state (GB1). Letters a-g denote various sites where segregation of isolated As atoms and As dimers were investigated. Results are listed in Table I.

axis. Figure 2 shows a second low-energy structure, we label GB2, with a total energy that is higher by only $0.15 \mathrm{eV}$ per periodic segment of the grain boundary plane. The two 
structures differ in the relative $<001>$-shift of the two grains forming the boundary, by a/8 $-0.68 \AA$, and also in the nature of the dislocation cores comprising the boundaries. The cores of GB1, are of the pure edge type $(b-a / 2<110>)$, while the cores of GB2 with Burger's vector $\mathbf{b}-a / 2<101>$ have mixed screw and edge character.

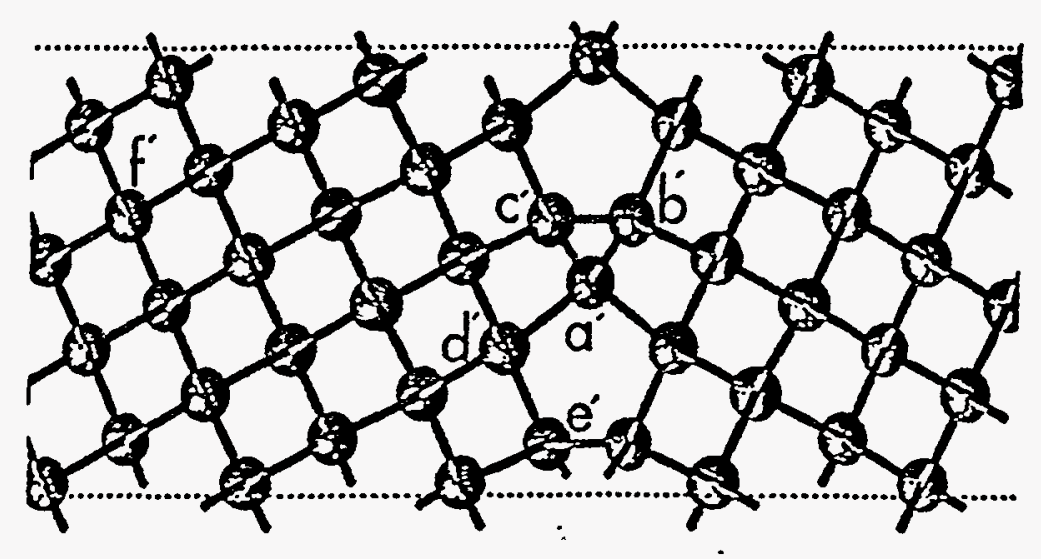

Fig. 2. Grain boundary of Fig. 1 in a metastable state (GB2) in the same projection view. Letters $a^{\prime}-f^{\prime}$ denote various sites at which segregation of isolated As atoms and As dimers were investigated. Segregation energies are listed in Table II.

We studied segregation of As atoms in both GB1 and GB2 because the two structures contain different dislocation cores that are components of many different grain boundaries. Figures 1 and 2 show labels for the sites where As atoms were placed: [a] through [e] are sites in the GB1 grain boundary whereas [f] and [g] are sites in the bulk; $\left[\mathrm{a}^{\prime}\right]$ through $\left[\mathrm{f}^{\prime}\right]$ denote corresponding sites for GB2. Symmetry has been used to reduce the number of possible distinct As sites and site pairs. Thus, using the symbol ' $\sim$ ' to indicate symmetry equivalence, we have for GBl: $a \sim c$ and $\mathrm{d} \sim \mathrm{e}$. It follows that for GB1 the possible distinct sites for atom segregation are [a] ([c]), [b], and [d] ([e]). The distinct site pairs for dimer segregation are $[a, c],[a, d],[b, c]$ and $[d, e]$. In addition, we have also studied the dimer $[f, g]$ where two atoms are placed at nearest-neighbor sites in the bulk crystal. For GB2 we have the equivalence $b^{\prime} \sim c^{\prime} \sim e^{\prime}$. The resulting distinct site pairs are $\left[a^{\prime}, d^{\prime}\right],\left[d^{\prime}, e^{\prime}\right],\left[b^{\prime}, c^{\prime}\right]$ and $\left[a^{\prime}, c^{\prime}\right]$.

Isolated As atoms and dimers

The results for isolated As substitutionals are shown in the top halves of Tables I and II. We find that all sites except [a] ([c]) on GB1 and [a'] on GB2 have a binding energy of $\sim 0.1 \mathrm{eV}$, the same value obtained for isolated As atoms in a similar boundary in Ge [8]. The smaller binding of [a] can be explained from the similarity of its environment, characterized by the surrounding bond-length and bond-angle distribution, to that of a bulk site [f] or [g], while an opposite effect occurs for [a']. In all cases of isolated As substitutionals, the lattice is found to undergo only a very small relaxation, in agreement with the results of Ref. 8.

We now turn to the dimer configurations. As we noted earlier, we performed calculations with both minimal and double periodicity in the grain-boundary plane, corresponding to dimer chains and isolated dimers, respectively. The latter calculations are extremely time consuming, and were, therefore, performed only for selected pairs of sites. We found that dimer formation in the grain boundary is energetically favored. If two As atoms are placed at neighboring substitutional sites in the bulk crystal, they repel each other seeking to achieve threefold coordination (pair [f, g] in Table I). The 
equilibrium As-As distance is $2.71 \AA$ compared to a Si-Si bond-length of $2.35 \AA$. The overall energy goes up by a tiny amount ( $0.01 \mathrm{eV}$ per $\mathrm{As}$ atom) as compared with isolated substitutional atoms because of the elastic energy cost. In contrast, As dimers in the grain boundary lead to an overall lowering of the energy. The selected calculations we performed for the isolated dimers yielded net binding of order $0.05-0.2 \mathrm{eV} / \mathrm{atom}$, indicating that the elastic energy cost in the grain boundary can be smaller than in the bulk. Dimer formation in the grain boundary is the result of repulsion between neighboring As atoms and occurs because this repulsion can be accommodated easier in the grain boundary than in the bulk crystal.

Table I. Segregation energies of single As atoms and As dimers placed substitutionally at various sites of a $\Sigma=5\{310\}<001>$ tilt boundary in Si with the GB1 structure (Fig. 1). For the dimer geometries the relaxed distance between the two dimer companion atoms (dAs-As) and the comesponding distance between the first As atom and the periodic image of its dimer companion ( $d_{A s-A s}$ ) are also indicated. See Fig. 1 for site denominations.

\begin{tabular}{|c|c|c|c|}
\hline $\begin{array}{c}\text { As } \\
\text { site(s) }\end{array}$ & $\begin{array}{c}\text { Segregation Energy } \\
\text { (eV/As atom) }\end{array}$ & $\begin{array}{c}\mathrm{d}_{\text {As-As }}(\hat{A}) \\
\text { (dimer geometries) }\end{array}$ & $\begin{array}{c}\mathrm{d}_{\text {As-As* }}(\AA) \\
\text { (dimer geometries) }\end{array}$ \\
\hline \hline$[\mathrm{f}]$ & 0.00 & & \\
{$[\mathrm{a}],[\mathrm{c}]$} & 0.03 & & \\
{$[\mathrm{~b}]$} & 0.11 & & \\
{$[\mathrm{~d}],[\mathrm{e}]$} & 0.11 & & 4.59 \\
{$[\mathrm{f}, \mathrm{g}]$} & -0.01 & 2.71 & 5.20 \\
{$[\mathrm{a}, \mathrm{d}]$} & 0.10 & 2.71 & 4.70 \\
{$[\mathrm{~d}, \mathrm{e}]$} & 0.19 & 2.79 & 3.76 \\
{$[\mathrm{a}, \mathrm{c}]$} & 0.20 & 2.89 & 3.43 \\
{$[\mathrm{~b}, \mathrm{c}]$} & 0.32 & 3.43 & \\
\hline
\end{tabular}

Table II. Lists the same information as Table I for the GB2 structure of a $\Sigma=5\{310\}<001>$ tilt boundary in Si. See Fig. 2 for site denominations.

\begin{tabular}{|c|c|c|c|}
\hline As site(s) & $\begin{array}{c}\text { Segregation Energy } \\
\text { (eV/As atom) }\end{array}$ & $\begin{array}{c}\mathrm{d}_{\mathrm{As}-\mathrm{As}}(\mathrm{A}) \\
\text { (dimer geometries) }\end{array}$ & $\begin{array}{c}\mathrm{d}_{\mathrm{As}-\mathrm{As}}(\mathrm{A}) \\
\text { (dimer geometries) }\end{array}$ \\
\hline \hline$\left[\mathrm{f}^{\prime}\right]$ & 0.00 & & \\
{$\left[\mathrm{~b}^{\prime}\right],\left[\mathrm{c}^{\prime}\right],\left[\mathrm{e}^{\prime}\right]$} & 0.12 & & \\
{$\left[\mathrm{~d}^{\prime}\right]$} & 0.13 & & \\
{$\left[\mathrm{a}^{\prime}\right]$} & 0.22 & & \\
{$\left[\mathrm{a}^{\prime}, \mathrm{d}^{\prime}\right]$} & 0.08 & 2.43 & 5.25 \\
{$\left[\mathrm{~d}^{\prime}, \mathrm{e}^{\prime}\right]$} & 0.09 & 2.72 & 4.84 \\
{$\left[\mathrm{~b}^{\prime}, \mathrm{c}^{\prime}\right]$} & 0.11 & 2.42 & 4.29 \\
{$\left[\mathrm{a}^{\prime}, \mathrm{c}^{\prime}\right]$} & 0.52 & 2.76 & 3.54 \\
\hline
\end{tabular}


The results for chains of As dimers are even more dramatic and are displayed in detail in Tables I and II for the two grain-boundary structures, respectively. We see that segregation energies range from 0.1 to $0.5 \mathrm{eV} /$ atom, the latter being in agreement with the measured values [1-3]. In tables I and II, the third column contains the As-As distance in the dimer, which is to be compared with the normal Si-Si distance of $2.35 \AA$. The fourth column contains the distance between an As atom and the periodic image of its dimer companion (As*).

We note three classes of results: (i) cases where the As-As* distance is significantly larger than the As-As distance, suggesting that the dimers in the chain are fairly well-separated. The segregation energy is small, less than $0.2 \mathrm{eV} /$ atom, comparable to that of truly isolated dimers that we discussed earlier; (ii) cases where the As-As and As-As* distances are comparable but different (e.g. [a, c] in GB1 and $\left[a^{\prime}, c^{\prime}\right]$ in GB2) where the segregation energy ranges from 0.2 to $0.5 \mathrm{eV} / \mathrm{atom}$; and (iii) a case where the As-As and As-As* distances are identical ([b, c] in GB1), corresponding to a fully ordered chain of As atoms, with an intermediate segregation energy of $0.32 \mathrm{eV} /$ atom.

An examination of the local three-dimensional topologies corresponding to Figures 1 and 2 suggests that the most stable dimer geometries in a chain are the ones in which one or both of the component As atoms can relax into the dislocation cores, where the average atomic density is lower than the crystalline bulk. Such relaxation reduces the strain in the Si backbonds, thereby yielding a larger segregation energy. It is interesting to note that the most stable chain of dimers $\left[a^{\prime}, c^{\prime}\right]$ in $\mathrm{GB} 2$ has its component atoms on two different dislocation cores, a configuration possible only in relatively-large-angle grain boundaries, while the chain of dimers $[b, c]$ on GBI has only one of its atoms (c) lying on a dislocation core, and could occur in any dislocation core of the perfect edge type.

\section{Possibility of Structural Phase Transition}

It is particularly interesting to note that the highest segregation energy in GB2 is significantly larger than in GB1, and might actually drive a structural transformation. In other words, the injection of a high concentration of As into polycrystalline Si may convert a grain boundary with perfect edge dislocations into one with mixed dislocations. Similar solute-induced grain boundary transformations have long been known to occur in metals [18], but we are not aware of any prior reports in semiconductors. From the relative total energies of the two grain boundaries we estimate that the transformation from GB1 to GB2 would require a critical As concentration of $\sim 19 \%$ in the column of the favored dimer sites. However, such a transformation involves a relative shift (sliding) of the two grains at the boundary, and the above estimate does not take into account any elastic energy cost that may be required to maintain integrity at triple junctions during the sliding process.

\section{SEGREGATION AT AN ISOLATED DISLOCATION CORE}

In the above section we studied the segregation of As at a high-angle grain boundary. For plastically deformed materials, as well as in low-angle grain boundaries the primary extended defects are dislocations [19]. In semiconductor devices, dislocations can severely affect the behavior of dopant impurities because: (1) the dislocation cores may provide a fast diffusion pathway to dopant impurities, which severely influences the dopant profile in an uncontrolled way, and (2) impurities may get trapped in the core regions leading to preferential segregation and electrical deactivation of the impurity. It 
is, therefore, important to investigate dopant segregation at an isolated dislocation core, and it is interesting to compare and contrast with segregation at a high-angle grain boundary. For concreteness, we chose a $90^{\circ}$ partial, a commonly occurring dislocation in Si.

\section{Relaxed Structures}

We used a noncubic periodic supercell with two oppositely oriented $90^{\circ}$ partial cores separated by a distance of 13 A [20] (Fig. 3). Previous studies found two models of reconstruction in the $90^{\circ}$ partial dislocation core: (1) the asymmetric reconstruction, in

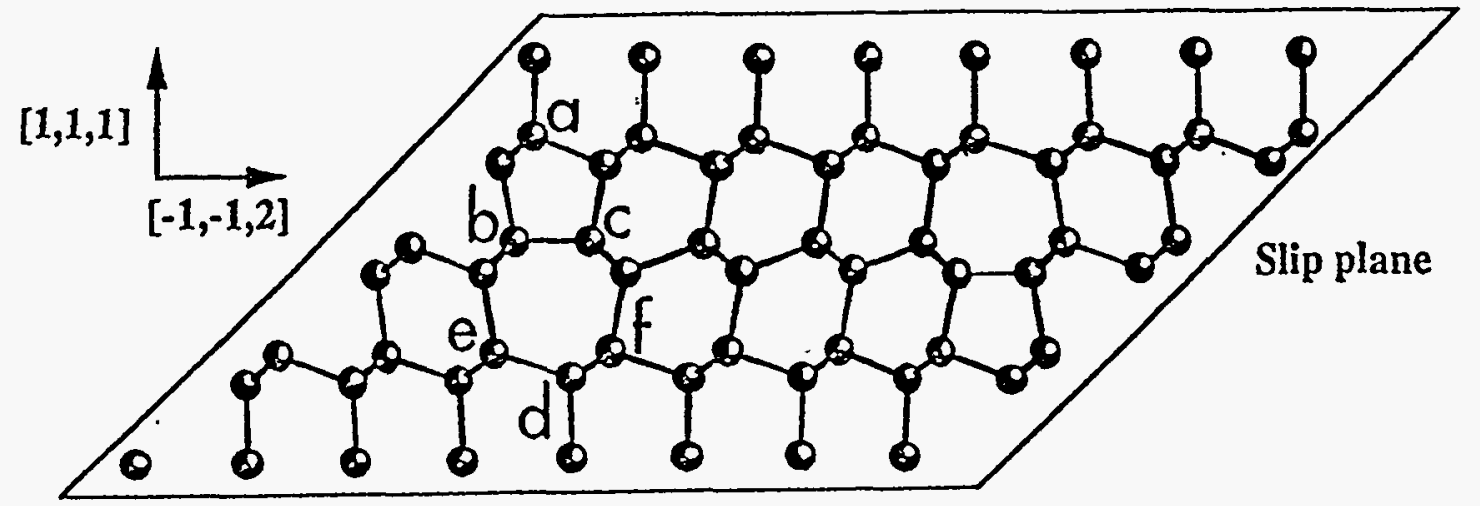

Fig. 3. Various sites of an isolated $90^{\circ}$ partial core, viewed along the dislocation line, on which single As atoms and As dimers are placed. Segregation energies are listed in Table III.

which the mirror symmetry along the dislocation line is broken $[21,22]$; and (2) the symmetric reconstruction, in which the mirror symmetry is kept intact [23]. The asymmetric stricture has all the atoms fourfold-coordinated, while the symmetric structure has two quasi fivefold-coordinated atoms per periodic segment of the core. Both these reconstructed cores look almost the same in the projection along the dislocation line (Fig. 3), but are clearly distinguishable in the bonding structures in the slip plane. From $a b$ initio relaxations we found that the asymmetric structure was stable, while the symmetric structure spontaneously transformed into the asymmetric structure, in agreement with an earlier $a b$ initio study [24]. All our segregation studies were thus performed exclusively on the asymmetric structure by substitutionally placing single As atoms and As dimers at various sites and nearest neighbor site pairs of the dislocation core, as indicated in Fig. 3.

\section{Isolated As atoms}

Let us first consider the single As atoms in the dislocation core. Four different sites were chosen. The strain distribution is very different at different sites, as is rapparent from comparing the site-associated bond-lengths with the bulk Si-Si bond-length of 2.35 $A$, making the segregation energy strongly site-dependent. Thus the strain is: (i) compressive at site a, with bond-lengths $(2.29,2.31,2.31,2.34) \AA$; (ii) tensile at site $d$, with bond-lengths $(2.37,2.41,2.43,2.44) \AA$; (iii) mixed compressive/tensile at sites $b$ and $c$ with bond-lengths $(2.31,2.34,2.41,2.43)$ A. The resulting segregation energy for As, listed in Table III, is largest for sites $b$ and $c$, moderate but positive at site $a$, and even negative for site d. The largest segregation energy of $0.33 \mathrm{eV}$ (sites $b, c$ ) is much larger than the average binding at a grain boundary $[8,10]$. Also, the wide variation of 
segregation energy from site to site is to be contrasted with a much smaller dispersion in a grain boundary [10], where the strain distribution is much more uniform. In all cases of single substitutional As, the relaxation from the initial Si site is very small, just as in a high-angle grain boundary.

\section{Dimer Chains}

Segregation studies of periodic chains of As dimers yield more dramatic results. To interpret these results, it should be re-emphasized that two As atoms placed on nearest neighbor Si sites, which we call As dimers, seek to repel and relax away from each other. In this way, each As atom achieves its preferred threefold coordination, leaving all the $\mathrm{Si}$ atoms fourfold-coordinated [10]. The three largest bonds in the dislocation core, i.e. [d, $f],[d, e]$ and $[b, c]$ were chosen for investigation. Table III displays the segregation energies for these three dimers, and the relaxed As-As and the As-As* distances. The distinct behaviors of the three dimers are clearly evident from Table III, as discussed below.

Table III. Lists the same information as Table I for As atoms and dimers segregated at the asymmetric structure of an isolated $90^{\circ}$ partial dislocation core in Si. See Fig. 3 for site denominations.

\begin{tabular}{|c|c|c|c|}
\hline As site(s) & $\begin{array}{c}\text { Segregation Energy } \\
\text { (eV/As atom) }\end{array}$ & $\begin{array}{c}\mathrm{d}_{\text {As-As }}(\hat{A}) \\
\text { (dimer geometries) }\end{array}$ & $\begin{array}{c}\mathrm{d}_{\text {As-As* }(\hat{A})} \\
\text { (dimer geometries) }\end{array}$ \\
\hline \hline bulk & 0.00 & & \\
[d] & -0.15 & & \\
[a] & 0.14 & & 2.42 \\
{$[\mathrm{~b}],[\mathrm{c}]$} & 0.33 & 2.57 & 4.53 \\
{$[\mathrm{~d}, \mathrm{f}]$} & 0.01 & 2.90 & 2.95 \\
{$[\mathrm{~d}, \mathrm{e}]$} & 0.13 & 2.87 & \\
{$[\mathrm{~b}, \mathrm{c}]$} & 0.88 & & \\
\hline
\end{tabular}

(i) $[d, f]$ : In this case the orientation of the dimer is such that site $d$ is the nearest neighbor of both $f$ and its nearest periodic image $f *$ in the dislocation line direction. Thus, in the periodic chain of dimers, all the As atoms are too close to each other. This allows only a small stretching of the As-As separation, to only $2.57 \AA$, while the As-As* distance $(2.42 \mathrm{~A}$ ) changes very little from the equilibrium Si-Si bond distance of $2.35 \mathrm{~A}$. Consequently, the relaxed structure consists of a chain of essentially fourfold-coordinated As atoms, resulting in negligible binding.

(ii) [d, e]: In this case the orientation of the dimer is nearly perpendicular to the dislocation line direction, and sites $\mathrm{d}$ and $\mathrm{e}^{*}$ are only second neighbors. This allows the As dimer atoms to relax away from each other, stretching the As-As separation to 2.90 $\AA$, thereby leading to threefold coordination of each As atom. However, the transverse orientation of the dimer keeps it well separated from its periodic images in the dislocation line direction, as evident from the large As-As* distance. We therefore have an array of nearly isolated As dimers. This leads to a positive but low segregation energy $(0.13 \mathrm{eV}$ per As), just as in a grain boundary [10].

(iii) $[b, c]$ : In this case the dimer is oriented such that although $b$ and $c^{*}$ are second nearest neighbors, their distance before relaxation is much less than the separation of $d$ and $e^{*}$ in case (ii). Consequently, the As-As stretch is accompanied by As and As* 
getting closer to each other. In the final relaxed geometry, the As-As* separation $(2.95 \AA)$ is only slightly larger than the As-As separation $(2.87 \mathrm{~A})$ and we have a nearly perfect chain of As atoms leading to a large segregation energy $(0.88 \mathrm{eV}$ per As).

\section{Electronic States}

In order to elucidate further the source of energy gain through the formation of dimer chains, we have examined the electronic energy levels in the As dimers in the dislocation core and compared them with those of isolated As atoms in the bulk. As one would expect, the isolated As atom has a shallow donor level at less than $0.1 \mathrm{eV}$ below the conduction band edge. In the $[\mathrm{b}, \mathrm{c}]$ dimer chains, on the other hand, each As atom has an electron at a level in the mid-gap region [25]. Further analysis indicates that the level in the gap is a member of the lone-pair states that As atoms have when they are threefoldcoordinated. Because of the close proximity of the threefold coordinated As atoms, these lone-pair states split, with half of them in the bandgap and half of them in the valence band. Thus, the gain in energy could be viewed as a result of the shallow donor level being driven deep into the bandgap by the lattice relaxation accompanying dimer formation, or, equivalently, as arising from the fact that dimer formation leads to lonepair states that are lower in energy than the states that are available when an As atom is fourfold-coordinated.

\section{DIRECT IMPURITY DETERMINATION WITH HIGH RESOLUTION STEM}

\section{Experiment}

The sample consisted of a silicon bicrystal wafer containing a $\Sigma=13\{510\}<001\rangle$ symmetric grain boundary. Plan view samples with a $<001>$ surface normal were characterized by high resolution Z-contrast imaging using a VG Microscopes HB603U scanning transmission electron microscope operating at $300 \mathrm{kV}$. The compositional sensitivity of the images, which is a function of the inner detector angle, can approach the atomic number $(Z)$ squared dependence of Rutherford scattering. With the resulting image, no preconceived model structures are required for image interpretation. The actual defect arrangements are apparent immediately [26]. Figure 4 displays a computer

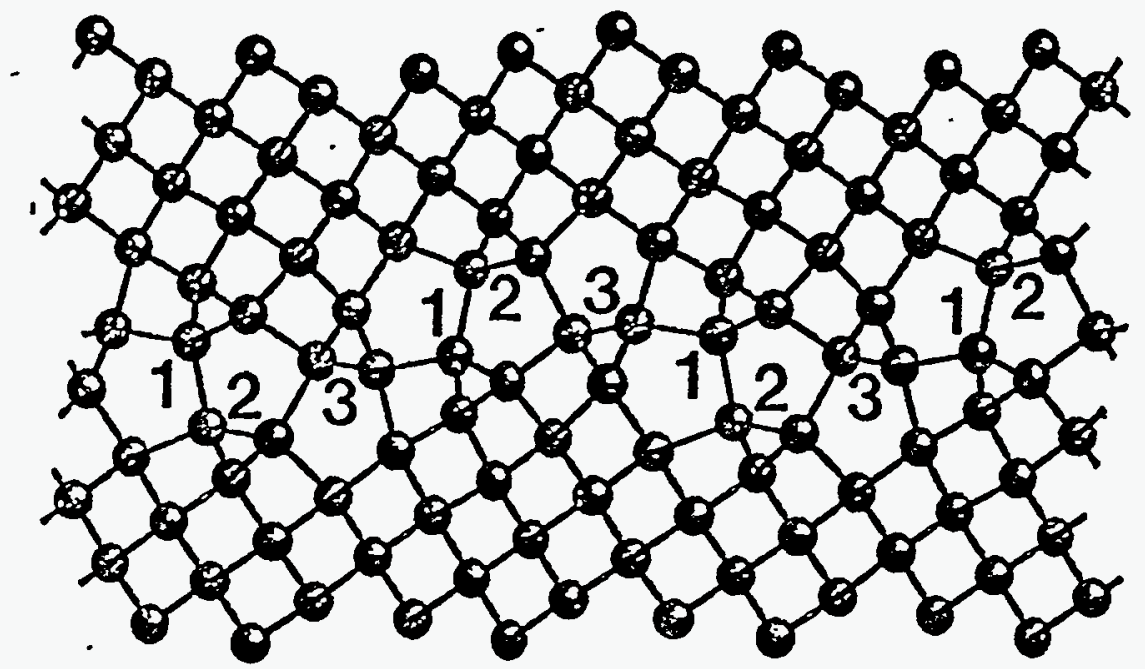

Fig. 4. Atomic model of a $\Sigma=13\{510\}<001>$ boundary imaged (Z-contrast) by STEM, viewed in a projection normal to the tilt axis. 
model of the atomic structure obtained from the Z-contrast image of the grain boundary in a projection normal to the tilt $(<001>)$ axis [27]. Each periodic segment consists of two equivalent sets of three triangles, denoted by 1,2,3 in Fig. 4.

The pure Si bicrystal was implanted at $450^{\circ} \mathrm{C}$ with arsenic ions $\left(450 \mathrm{keV}, 3 \times 10^{16}\right.$ ions $/ \mathrm{cm}^{2}$ ). Subsequent to implantation, the wafer was annealed first for one week at $1000^{\circ} \mathrm{C}$ followed by one week at $700^{\circ} \mathrm{C}$. In the doped crystal, the average intensity from triangles 2 is $20 \%$ brighter than the average intensity from the triangles 1 or 3 [28]. These bright features, which are periodically repeated every $0.69 \mathrm{~nm}$, are not observed in the undoped bicrystal. From careful intensity analysis, we have estimated an As concentration of $\sim 5-6 \%$ in the bright triangles and $\sim 1-2 \%$ or less in the dark triangles, respectively.

\section{Theoretical Studies}

In order to explain why triangles 2 light up specifically, we have performed $a b$ initio calculations for segregation of As in a $\Sigma=13\{510\}<001>$ boundary in $\mathrm{Si}$, similar to that described in the previous two sections for a $\Sigma=5$ boundary and a $90^{\circ}$ partial dislocation core. Due to constraints imposed by heavy computational requirements, we used the minimum periodic repeat lengths in the grain boundary plane, i.e. $0.54 \mathrm{~nm}$ and $1.38 \mathrm{~nm}$ along and perpendicular to the tilt axis, respectively. In a direction normal to the grain boundary plane, we used two oppositely oriented grain boundaries separated by $2.94 \mathrm{~nm}$.

The calculations indicate that for isolated substitutional As atoms the segregation energies vary somewhat from site to site, but is always around $0.1 \mathrm{eV}$, irrespective of the location. The result is similar to earlier results for the $\Sigma=5$ boundary in $S i$ [10] and $\mathrm{Ge}$ [8]. This result clearly implies that the higher intensity observed in triangles 2 must be due to arsenic segregation in some form other than as isolated substitutional atoms.

Guided by the segregation studies described earlier, the obvious thing to look for was the segregation of dimers. Indeed, dimer chains yielded threefold coordinated As and larger segregation energies. However, estimates from our intensity analysis implied dilute As limit. Assuming that equilibrium had been established on and around the grain boundary, statistical mechanics can be used to show that, in the dilute limit, dopants - segregate either as isolated atoms or more strongly as isolated dimers. The formation of chains of two or more dimers are entropically almost precluded. By a straightforward maximization of the partition function, the concentration of As as isolated As atoms $\left(n_{1}\right)$ and isolated As dimers $\left(n_{2}\right)$ can be expressed in terms of the bulk As concentration ( $\left.n_{B}\right)$ as:

and

$$
n_{1}=\frac{n_{B}}{1-n_{B}} \exp \left(\Delta_{1} / k_{B} T\right),
$$

$$
\mathrm{n}_{2}=2\left(\frac{\mathrm{n}_{\mathrm{B}}}{1-\mathrm{n}_{\mathrm{B}}}\right)^{2} \exp \left(2 \Delta_{2} / \mathrm{k}_{\mathrm{B}} \mathrm{T}\right),
$$

where, $\Delta_{1}$ and $\Delta_{2}$ are the segregation energies per As atom for the isolated atom and isolated dimer respectively, $T$ is the equilibrium temperature, and $k_{B}$ the Boltzmann constant. From $a b$ initio calculations we estimated $\Delta_{1} \sim 0.1 \mathrm{eV}$ for all triangles, and $\Delta_{2} \sim$ $0.3 \mathrm{eV}$ and $\sim 0.1 \mathrm{eV}$ respectively for the bright $(2)$ and dark $(1,3)$ triangles. Assuming an equilibrium temperature of $700^{\circ} \mathrm{C}$ and a bulk As concentration of $\sim 0.4 \%$ (close to the heavy doping limit of As in crystalline $\mathrm{Si}$ ), the total As concentration $\left(\mathrm{n}_{\mathrm{G}}=\mathrm{n}_{1}+\mathrm{n}_{2}\right.$ ) given by eqs. (1) and (2) is $\sim 5 \%$ in the bright triangles and $\sim 1.5 \%$ in the dark triangles, in agreement with estimates from the intensity analysis of our Z-contrast images. Thus, 
our STEM observations and calculations strongly indicate that As and presumably other n-type dopants (e.g. P) can attain their preferred threefold-coordination in the cores of dislocations and grain boundaries without forcing neighboring Si atoms to become overor under-coordinated [28].

\section{SUMMARY}

In summary, cooperative phenomena involving chains of threefold-coordinated As atoms or dimers result in much larger segregation energies than isolated As substitutionals, both in high-angle grain boundaries and in isolated dislocation cores. Segregation energies, thus obtained for a $\Sigma=5$ tilt boundary, are in agreement with experimental values for As segregation in poly-Si. This provides a mechanism for As segregation that does not require the presence of steps or other defects. Dimer chains in mixed dislocation cores are found to have lower energies, raising the intriguing possibility of segregation-induced structural transformation in grain boundaries containing pure edge dislocations. The overall behavior of single impurities, isolated dimers, and chains in an isolated dislocation core is similar to the behavior in a highangle grain boundary. However, the segregation energy of both single As atoms and those in a chain are much larger in an isolated dislocation core, with the result for the chain being as high as $0.9 \mathrm{eV}$ per As atom. Finally, high resolution STEM imaging of a $\Sigma=13$ tilt boundary in Si doped with As have conclusively shown that As segregates preferentially only to certain dislocations (triangles) within the grain boundary core, which can be explained by the formation of As dimers.

\section{ACKNOWLEDGMENTS}

We would like to thank the ORNL Center for Computational Sciences for providing valuable supercomputer hours. This. research was supported in part by Lockheed Martin Energy Research Corp. under DOE contract No. DE-AC0596OR22464, and ONR grant No. N00014-95-1-0906, and by an appointment to the ORNL Postdoctoral Research Associates Program administered jointly by ORNL and ORISE.

\section{REFERENCES}

1. C. R. M. Grovenor, J. Phys. C 18, 4079 (1985).

2. C. R. M. Grovenor, P. E. Batson, D. A. Smith, and C. Y. Wang, Phil. Mag. A 50, 409 (1984).

3. M. M. Mandurah, K. C. Saraswat, C. R. Helms, and T. I. Kamins, J. Appl. Phys. 51, 5755 (1980).

4. J.-L. Maurice, in Polycrystalline Semiconductors II, edited by J. H. Werner and H. P. Strunk, Springer Proceedings in Physics Vol. 54 (Springer, Berlin, 1992), p. 166.

5. A. Bourret and J. L. Rouvière, Springer Proc. Phys. 35, 8 (1989).

6. M. Kohyama, Phys. Stat. Sol. (b) 141, 71 (1987). 
7. A. T. Paxton and A. P Sutton, J. Phys. C 21, L481 (1988).

8. T. A. Arias and J. D. Joannopoulos, Phys. Rev. B 49, 4525 (1994).

9. N. F. Mott, Adv. Phys. 16, 49 (1967).

10. A. Maiti, M. F. Chisholm, S. J. Pennycook, and S. T. Pantelides, Phys. Rev. Lett. 77, 1306 (1996).

11. P. Hohenberg and W. Kohn, Phys. Rev. 136, B864 (1964).

12. J. P. Perdew and A. Zunger, Phys. Rev. B 23, 5048 (1981).

13. G. P. Kerker, J. Phys. C 13, L189 (1980).

14. L. Kleinmann and D. M. Bylander, Phys. Rev. Lett. 48, 1425 (1982).

15. R. D. King-Smith, M. C. Payne, and J. S. Lin, Phys. Rev. B 44, 13063 (1991).

16. H. J. Monkhorst and J. D. Pack, Phys. Rev. B 13, 5188 (1976).

17. M. C. Payne, M. P. Teter, D. C. Allan, T. A. Arias, and J. D. Joannopoulos, Rev. Mod. Phys. 64, 1045 (1992).

18. V. Vitek and G. J. Wang, Surf. Sci. 144, 110 (1984); K. E. Sickafus and S. L. Sass, Acta Met. 35, 69 (1987); C. Rottman, J. de Phys. 49, C5, 313 (1988).

19. J. P. Hirth and J. Lothe, Theory of Dislocations (McGraw-Hill, New York, 1982).

20. A. Maiti, T. Kaplan. M. Mostoller, M. F. Chisholm, S. J. Pennycook, and S. T. Pantelides, Appl. Phys. Lett., tentative Jan. 20 (1997).

21. R. Jones, J. Phys. (Paris), Colloq. 40; C6-33 (1979).

22. P. B. Hirsch, J. Phys. (Paris), Colloq. 40, C6-27 (1979).

23. M. S. Duesbery, B. Joos, and D. J. Michel, Phys. Rev. B 43, 5143 (1991).

24. J. R. K. Bigger, D. A. McInnes, A. P. Sutton, M. C. Payne, I. Stich, R. D. KingSmith, D. M. Bird, and L. J. Clarke, Phys. Rev. Lett. 69, 2224 (1992).

25. In the local density approximation used in the calculations, the bandgap is smaller than the experimental bandgap, so we cannot unambiguously determine the precise location of the level relative to the conduction band edge. Note that, however, for total-energy calculations performed here, only the occupied states are pertinent.

26. D. E. Jesson and S. J. Pennycook, Proc. R. Soc. Lond. A 449, 273 (1995).

27. M. F. Chisholm, M. Mostoller, T. Kaplan, and S. J. Pennycook, Phil. Mag. A, in press.

28. M. F. Chisholm, A. Mait, S. J. Pennycook, and S. T. Pantelides, Science, submitted. 